Please do not remove this page

RMIT

UNIVERSITY

\title{
Integrated tunable microfluidic interferometer
}

Monat, Christelle; Domachuk, Peter; Grillet, Christian; Eggleton, Ben; Mutzenich, Simon; Rosengarten, Gary; Mitchell, Arnan

https://researchrepository.rmit.edu.au/esploro/outputs/9921862772601341/filesAndLinks?institution=61RMIT_INST\&index=null

Monat, C., Domachuk, P., Grillet, C., Eggleton, B., Mutzenich, S., Rosengarten, G., \& Mitchell, A. (2006).

Integrated tunable microfluidic interferometer. Proceedings of the 31st Australian Conference on Optical Fibre Technology and Australian Optical Society (ACOFT\&AOS 2006), 54-56.

https://doi.org/10.1109/ACOFT.2006.4519294

Published Version: https://doi.org/10.1109/ACOFT.2006.4519294

Repository homepage: https://researchrepository.rmit.edu.au

(C) 2006 IEEE

Downloaded On 2023/04/27 01:00:08 +1000

Please do not remove this page 
Citation: Monat, C, Domachuk, P, Grillet, C, Eggleton, B, Mutzenich, S, Rosengarten, G and Mitchell, A 2006,

'Integrated tunable microfluidic interferometer', in Proceedings of the 31st Australian Conference on Optical Fibre Technology and Australian Optical Society (ACOFT and AOS 2006), Melbourne, Australia, $10-13$ July 2006. ACOFT/AOS 2006 - Proceedings

Melbourne, Australia, 10 - 13 July 2006

\title{
Integrated Tunable Microfluidic Interferometer
}

\author{
Christelle Monat, Peter Domachuk, Christian Grillet, Benjamin J. Eggleton \\ CUDOS Research Group, School of Physics, University of Sydney, NSW, 2006, Australia \\ Simon Mutzenich, Gary Rosengarten, Arnan Mitchell \\ MMTC, School of Electrical and Computer Engineering, RMIT University, VIC 3001, Australia
}

\begin{abstract}
We demonstrate a tunable optical filter/sensor based on a microfluidic interferometer integrated onto a compact planar chip. The interaction of the beam with a water/air interface provides a tunable Mach-Zehnder response with $28 \mathrm{~dB}$ extinction ratio.
\end{abstract}

\section{INTRODUCTION}

The next generation of photonic devices for optical communications and sensing should display two key features: compactness for large scale integration and tunability for an increased degree of functionality. However, the traditional electro-optic and thermo-optic tuning mechanisms lack the range of refractive index modulation required to achieve sufficient photonic tunability on the micrometer scale.

Microfluidics, developed originally for ink jet printing and later biotechnology, appears for several reasons as a promising solution for tuning optical devices at the micrometer scale [1-4]. First, the refractive index contrast achievable between a fluid and the surrounding air is large, which enables appropriate phase delays for interferometric devices in a compact space. Second, fluid is a mobile phase which allows for flexible and significant refractive index changes through simple motion of a fluid body: the key towards tunability. To produce ultimately compact and practical devices based on this hybrid technology, both microfluidics and microphotonic functionalities should be integrated onto one single planar chip. Recently, we reported a tunable filter based on a compact microfluidic interferometer [5]. Although good performance was achieved this particular demonstration was not fully integrated unlike other designs [6-7].

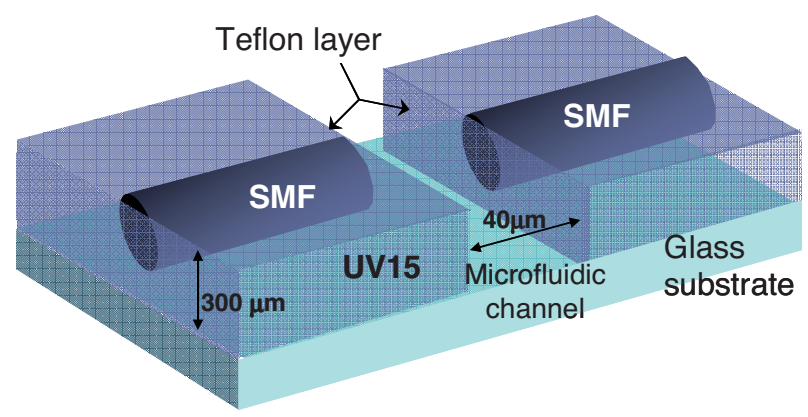

Fig. 1. A schematic of the whole integrated device, consisting in two self aligned buried SMF separated by a microfluidic channel.
In this paper we demonstrate a compact microfluidic optical interferometer completely integrated onto a single planar substrate. The device fabrication overcomes any critical alignment between the input and output light paths since it uses a buried single mode fiber (SMF) sectioned by a microfluidic channel (Fig. 1). The interferometer uses a fluid/air interface, the meniscus, which provides a sharp refractive index discontinuity (Fig. 2) which is placed in the middle of the optical beam. The resulting interaction produces a Mach-Zehnder-like response, where the visibility of the periodic spectral response can be finely adjusted by varying the position of the meniscus. The results are consistent with beam propagation method (BPM) simulation. As the meniscus shape has proved to be a crucial parameter of the device [1], an important innovation of this work is that a sputtered thin Teflon layer is coated onto the microfluidic channel to achieve a flattened meniscus. This results in enhanced performance of the device which displays an extinction ratio of $28 \mathrm{~dB}$. Finally, tunability of the spectral response is demonstrated through the actuation of the meniscus over less than $2 \mu \mathrm{m}$ around the beam center.

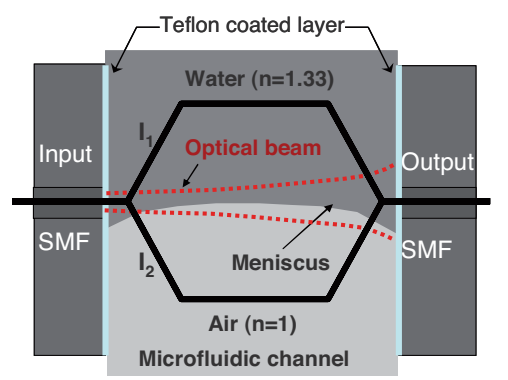

Fig 2. A top schematic representation of the single beam fluid Mach-Zehnder interferometer, with the path through the channel highlighted in red, superimposed on a schematic representation of a traditional Mach Zehnder interferometer, where $\mathrm{I}_{1}$ and $\mathrm{I}_{2}$ are the two split and delayed beams.

\section{DESIGN AND FABRICATION OF THE INTERFEROMETER}

Fig. 1 shows a schematic of the interferometer. A SMF is first buried in cured UV15 photopolymer. A dicing saw is then used to open a $40 \mu \mathrm{m}$ wide straight channel perpendicular to the SMF. At the same time the saw bisects the SMF, leaving two remaining self-aligned SMF separated by a microfluidic channel. After a careful top polishing, the device is Teflon plasma coated so as to deposit a conformal 
$300 \mathrm{~nm}$ thick layer, which aims to change the otherwise hydrophilic surface of the channel. The latter is sealed and roughly aligned with a top PDMS layer, in which holes are drilled for fluid insertion. Deionized water (1.33 refractive index) is introduced with a syringe pump into the channel and can be easily moved in front of the fibers (Fig. 3a, 3b, 3c). The optical signal from a broadband white light is analyzed by an optical spectrum analyzer.

(a)

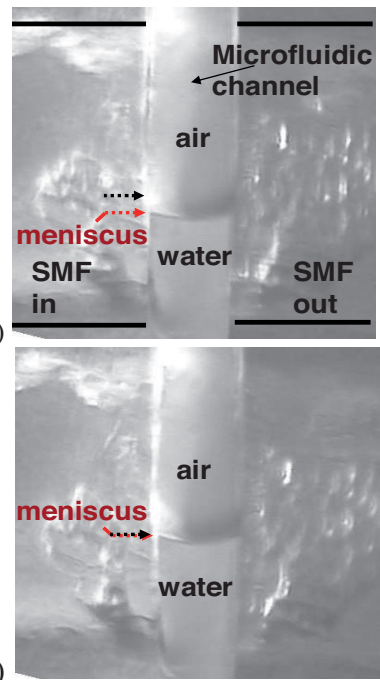

(c)

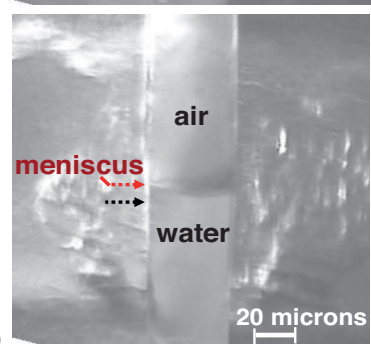

Fig. 3. Top view photographs from the final device, showing different locations of the air/water meniscus, while it moves in the upper direction across the channel (from top to bottom).

\section{RESULTS AND DISCUSSION}

In a classical interferometer, the incident light is divided into two parts, one of which undergoes some phase delay relative to the other one. Here, an optical path difference is induced between two sections of the same beam during its propagation across the meniscus (see Fig. 2). As those two sections propagate in different media presenting a refractive index difference, $\Delta \mathrm{n}$, along the microfluidic channel of width $\mathrm{h}$, they present a relative phase delay when recombining at the entrance of the filtering output SMF, creating a MachZehnder like response. This is easily described by a simple analytical equation [1]:

$$
I=I_{1}+I_{2}+2 \cos \left(\frac{2 \pi h \Delta n}{\lambda}\right) \sqrt{I_{1} I_{2}}
$$

where $I$ is the observed intensity at the free space wavelength $\lambda$, and $I_{1}$ and $I_{2}$ are the signal intensities that propagates in water and air, respectively. The transmission dip wavelengths and the associated free spectral range of the interferometer are related to the path difference induced between the two beams, depending on $h$ and $\Delta n$. Fig. 4 shows the experimental response of the device along with the transmission calculated from (1) $(h=40 \mu \mathrm{m}$ and $\Delta n=0.33)$ when the meniscus exactly sections the center of the beam. The exhibited strong extinction ratio $(\sim 28 \mathrm{~dB})$ as well as good agreement with the analytical model confirms the flatness of the meniscus.

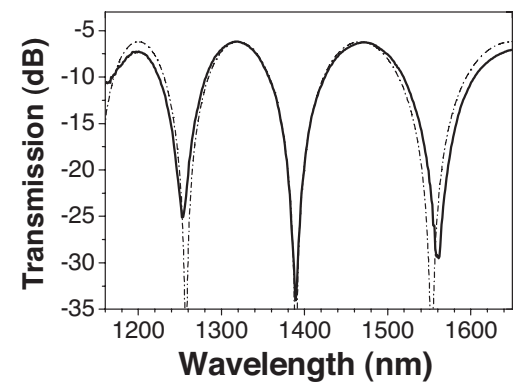

Fig. 4. Spectral response of the microfluidic Mach-Zehnder (solid line) along with the analytical model (dotted line)

When varying the meniscus location relative to the center of the beam, the strength of the dips is tuned as can be seen on Fig. 5a. Since the location of the meniscus defines the fraction of the beam which propagates through either air or water, it affects the contrast of the interferometer response by modifying the relative $I_{1}$ and $I_{2}$ signal intensity values in (1). BPM simulations, which take into account beam divergence, give consistent results as displayed on Fig. 5b, obtained by modeling a flat meniscus.
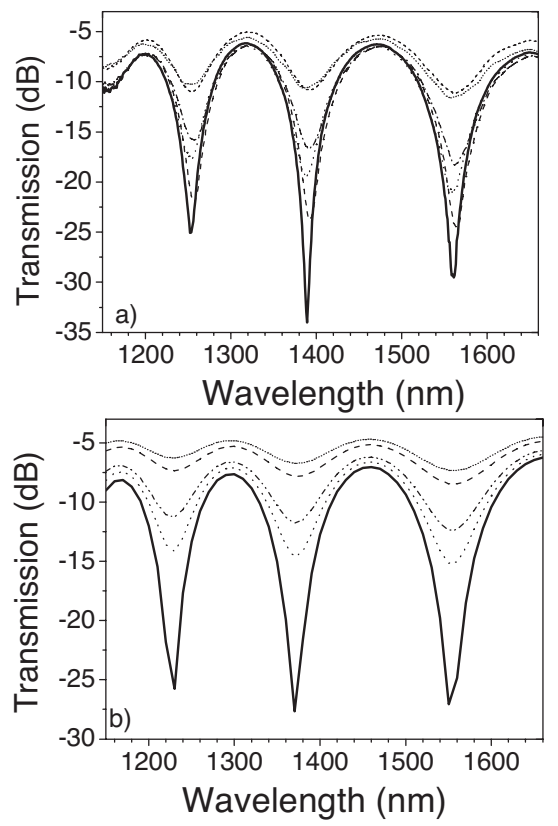

Fig. 5. Tuning of the spectral response obtained (a) experimentally and (b) with BPM simulations when moving the meniscus in front of the fibers. 
As compared to previous demonstrations, the device integration makes the incident light directly interact with the meniscus, enabling a strong extinction ratio as well as low expected insertion loss. The $5 \mathrm{~dB}$ displayed insertion loss can be actually attributed to the wide $(40 \mu \mathrm{m})$ microfluidic channel, which is responsible for a high fraction of the diverging beam to stand outside the output SMF acceptance angle. BPM simulation shows that a factor of 2 can be gained by decreasing the channel width down to $20 \mu \mathrm{m}$. Note that the insertion loss value of $5 \mathrm{~dB}$ further confirms the flatness of the meniscus: indeed, a curved meniscus would provide much larger insertion losses for the same microfluidic channel dimension.

The sensitivity of the interferometer response upon the meniscus location has been quantitatively measured, as displayed on Fig. 6. The contrast of the device response is plotted versus the location of the meniscus relative to the center of the SMF for both experiment and BPM simulation. The experimental results show good agreement with the BPM simulation and exhibit a strong variation for less than $2 \mu \mathrm{m}$ movement of the meniscus around the center of the SMF.

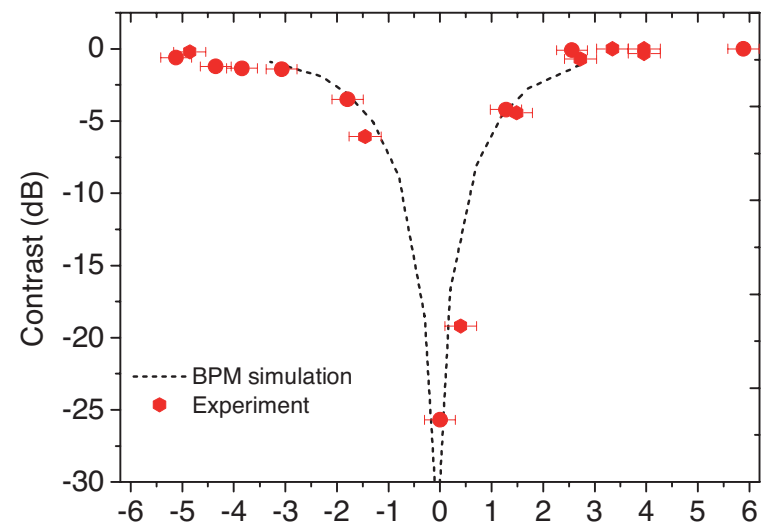

Location of the meniscus relative to the SMF center $(\mu \mathrm{m})$

Fig. 6. Variation of the contrast (difference between the dip transmission and the insertion loss) of the microfluidic interferometer response when the meniscus is moved in front of the SMF, for both experimental results (red points) and BPM simulation (dotted line).

As the device response has proved to be very sensitive to both the meniscus location and shape, it could be used like a highly sensitive, compact, ambient pressure sensor, the teflon coated channel providing an ideal environment for easily tuning the meniscus location upon external pressure gradient. Combined with microfluidic valves and electro-wetting techniques to tune the meniscus location [8], this new class of device has potentialities in bio sensing, medical diagnosis as well as telecommunications. Furthermore, since the response also depends on the fluid index, this device could be used for highly compact refractometry, while the wavelength of resonance could be tuned using an electro-optic liquid, such as liquid crystals.

\section{CONCLUSION}

We have demonstrated a compact, integrated microfluidic interferometer that utilizes the interaction of a single beam with a sharp air/water interface on a planar chip. The obtained Mach-Zehnder response displays a strong extinction ratio of $28 \mathrm{~dB}$, enabled by both the device integration and careful control of the meniscus shape through the teflon coating process. Good agreement of the experimental results has been obtained with both BPM and an analytical model, which proves the interface is sufficiently flat. The $5 \mathrm{~dB}$ insertion loss is mainly limited by the large width of the microfluidic channel and can be reduced by optimizing dimensions. Finally, tunability has been displayed by adjusting the location of the meniscus of less than $2 \mu \mathrm{m}$ in front of the beam.

This work was funded under ARC research grant DP0556781 (Microfluidic photonics).

\section{REFERENCES}

[1] C. Kerbage, and B. J. Eggleton, "Tunable microfluidic optical fiber gratings", Appl. Phys. Lett. vol. 82, no. 9, pp. 1338-1340 (2003).

[2] L. Zhu, Y. Huang, and A. Yariv, "Integrated microfluidic variable optical attenuator", Opt. Express vol. 13, no. 24, pp. 9916-9921 (2005)

[3] U. Levy, K. Campbell, A. Groisman, S. Mookherjea, and Y. Fainman, "On-chip microfluidic tuning of an optical microring resonator", Appl. Phys. Lett. vol. 88, no. 11, pp. 1111071-3 (2006).

[4] D. Erickson, T. Rockwood, T. Emery, A. Scherer, and D. Psaltis, "Nanofluidic tuning of photonic crystal circuits", Opt. Lett. vol. 31, no. 1, pp. 59-61 (2006).

[5] C. Grillet, P. Domachuk, V. Ta'eed, E. Magi, J. A. Bolger, B. J. Eggleton, L.E. Rodd, J. Cooper-White, "Compact tunable microfluidic interferometer", Opt. Express, vol. 12, no. 22, pp. 5440-5447 (2004).

[6] T. Mizuno, H. Takahashi, T. Kitoh, M. Oguma, T. Kominato, and T. Shibata, Proceedings ECOC 2004, vol. 2, pp. 144-145.

[7] G. P. Nordin, S. Kum, J. Cai, and J. Jiang, "Hybrid integration of conventional waveguide and photonic crystal structures", Opt. Express, vol. 10, no. 23, pp. 1334-1341 (2002).

[8] J. L. Jackel, S. Hackwood, J. J. Veselka, and G. Beni, "Electrowetting switch for multimode optical fibers", Applied Optics, vol.22, no. 11, pp. 1765-1770 (1983). 